\title{
Global Stability of an HIV-1 Infection Model with General Incidence Rate and Distributed Delays
}

\author{
Abdoul Samba Ndongo and Hamad Talibi Alaoui \\ Department of Mathematics and Informatics, Faculty of Sciences, Chouaib Doukkali University, BP 20, 24000 El Jadida, Morocco \\ Correspondence should be addressed to Abdoul Samba Ndongo; abdoulsambandongo@yahoo.fr
}

Received 6 March 2014; Accepted 8 July 2014; Published 29 October 2014

Academic Editor: Shengqiang Liu

Copyright ( 2014 A. S. Ndongo and H. Talibi Alaoui. This is an open access article distributed under the Creative Commons Attribution License, which permits unrestricted use, distribution, and reproduction in any medium, provided the original work is properly cited.

\begin{abstract}
In this work an HIV-1 infection model with nonlinear incidence rate and distributed intracellular delays and with humoral immunity is investigated. The disease transmission function is assumed to be governed by general incidence rate $f(T, V) V$. The intracellular delays describe the time between viral entry into a target cell and the production of new virus particles and the time between infection of a cell and the emission of viral particle. Lyapunov functionals are constructed and LaSalle invariant principle for delay differential equation is used to establish the global asymptotic stability of the infection-free equilibrium, infected equilibrium without $B$ cells response, and infected equilibrium with $B$ cells response. The results obtained show that the global dynamics of the system depend on both the properties of the general incidence function and the value of certain threshold parameters $R_{0}$ and $R_{1}$ which depends on the delays.
\end{abstract}

\section{Introduction}

Immunity can be broadly categorized into adaptive immunity and innate immunity. Adaptive immunity is mediated by clonally distributed $T$ and $B$ lymphocytes, namely, humoral and cellular immunity, and is characterized by specificity and memory. The humoral immunity plays an important role in antiviral defence by attacking virus. A basic mathematical model describing HIV-1 infection dynamics model with humoral immunity was introduced by Murase et al. [1] as

$$
\begin{gathered}
\frac{d T(t)}{d t}=\Lambda-d T(t)-\beta T(t) V(t), \\
\frac{d I(t)}{d t}=\beta T(t) V(t)-\delta I(t), \\
\frac{d V(t)}{d t}=N \delta I(t)-c V(t)-q B(t) V(t), \\
\frac{d B(t)}{d t}=g B(t) V(t)-\mu B(t),
\end{gathered}
$$

where $T(t), I(t), V(t)$, and $B(t)$ represent the densities of uninfected cells, infected cells, virus, and $B$ cells at time $t$, respectively. $\Lambda$ and $d$ are the birth and death rate constants of uninfected cells. $\beta$ is the infection rate, $N$ is the average number of virus particles produced over the lifetime of a single infected cells, and $\delta$ is the death rate of infected cells; $c$ is the death rate constant of the virus, $g$ and $\mu$ are the recruited rate and death rate constants of $B$ cells, and $q$ is the $B$ cells neutralization rate. Mathematical models for virus dynamics with antibody immune response has drawn much attention of researchers (see, e.g., [1-13] and the reference therein). Recently many studies have been done to improve the model (1) by introducing delays and changing the incidence rate according to different practical background. These studies used different delayed models with different forms of incidence rate; see, for example, [6, 9-11] for discrete delays and $[5,13]$ for distributed delays.

In the present paper, motivated by the works of $[1,5,13]$, we propose the following model with a general incidence rate and distributed delays and humoral immunity:

$$
\begin{aligned}
\frac{d T(t)}{d t}=\Lambda & -d T(t)-f(T(t), V(t)) V(t), \\
\frac{d I(t)}{d t}= & \int_{0}^{h_{1}} P_{1}(\tau) e^{-m_{1} \tau} f(T(t-\tau), V(t-\tau)) \\
& \times V(t-\tau) d \tau-\delta I(t),
\end{aligned}
$$




$$
\begin{gathered}
\frac{d V(t)}{d t}=N \delta \int_{0}^{h_{2}} P_{2}(\tau) e^{-m_{2} \tau} I(t-\tau) d \tau \\
-c V(t)-q B(t) V(t), \\
\frac{d B(t)}{d t}=g B(t) V(t)-\mu B(t),
\end{gathered}
$$

where the parameters in system (2) have the same meanings as in system (1). $f(T, V) V$ is the general incidence rate. It is assumed in (2) that the uninfected cells that are contacted by the virus particles at time $t-\tau$ become infected cells at time $t$, where $\tau$ is distributed according to $P_{1}(\tau)$ over the interval $\left[0, h_{1}\right]$, where $h_{1}$ is the limit superior of this delay. The constant $m_{1}\left(m_{1}>0\right)$ is assumed to be the death rate for infected cells during time period $[t-\tau, t]$ but not yet virus-producing cells and the term $e^{-m_{1} \tau}$ denotes the surviving rate of infected cells during the delay period. On the other hand, it is assumed in (2) that a cell infected at time $t-\tau$ starts to yield new infectious virus at time $t$, where $\tau$ is distributed according to a probability distribution $P_{2}(\tau)$ over the interval $\left[0, h_{2}\right]$ and $h_{2}$ is limit superior of this delay. The factor $e^{-m_{2} \tau}$ accounts for the probability of surviving infected cells during the time period of delay, where $m_{2}$ is constant. In (2), the probability distribution functions $P_{i}(\tau), i=1,2$, are assumed to satisfy $P_{i}(\tau)>0, i=1,2$ and

$$
\int_{0}^{h_{i}} P_{i}(\tau) d \tau=1, \quad \int_{0}^{h_{i}} \tau P_{i}(\tau) d \tau<\infty, \quad i=1,2 .
$$

The function $f$ is assumed to be continuously differentiable in the interior of $I R_{+}^{2}$ and satisfies the following hypotheses:

$$
\begin{aligned}
& \left(H_{1}\right) f(0, V)=0, \text { for all } V \geq 0, \\
& \left(H_{2}\right) \partial f(T, V) / \partial T>0, \text { for all } T>0 \text { and } V \geq 0, \\
& \left(H_{3}\right) \partial f(T, V) / \partial V \leq 0, \text { for all } T>0 \text { and } V \geq 0 . \\
& \left(H_{4}\right) \partial(f(T, V) V) / \partial V \geq 0, \text { for all } T, V>0 .
\end{aligned}
$$

The biological meaning of hypothesis $\left(H_{1}\right)$ to $\left(H_{4}\right)$ is given in [10].
Note the following.

The incidence rate $f(T, V) V$ given in (2) generalizes many common forms such as $[5,9,13]$ (see Section 6).

The distributed delay is more general than the discrete one and it is more adapted to biological phenomena.

$h_{1}$ or $h_{2}$ can be infinity.

The present paper is organized as follows. In Section 2, we establish the nonnegativity and boundedness of solutions and we derived the basic reproduction ratios for viral infection and humoral immune response $R_{0}$ and $R_{1}$, respectively. In Section 3, the existence of a possible three positive equilibria, an infection-free equilibrium $E_{0}^{*}$, an infected equilibrium without $B$ cells response $E_{1}^{*}$, and an infected equilibrium with $B$ cells response $E_{2}^{*}$, is established. In Sections 4 and 5 , we show that the global asymptotic stability of these equilibria depend only on the basic reproduction numbers under some hypotheses on the incidence function. In Section 6, some examples are given. A brief discussion is given in the last section to conclude this paper.

\section{Preliminary Results}

The initial conditions of (2) are given as

$$
\begin{gathered}
T(\theta)=\phi_{1}(\theta), \quad I(\theta)=\phi_{2}(\theta), \quad V(\theta)=\phi_{3}(\theta), \\
B(\theta)=\phi_{4}(\theta), \\
\phi_{i}(\theta) \geq 0, \quad i=1,2,3,4, \theta \in[-h, 0], \quad h=\max \left\{h_{1}, h_{2}\right\},
\end{gathered}
$$

where $\phi=\left(\phi_{1}, \phi_{2}, \phi_{3}, \phi_{4}\right) \in C^{+}$, here $C^{+}=C\left((-h, 0], \mathfrak{R}_{+}^{4}\right)$, with $C\left((-h, 0], \Re^{4}\right)$; denotes the Banach space of continuous functions mapping the interval $(-h, 0]$ into $\mathfrak{R}^{4}$.

Theorem 1. Under the initial conditions (4), all solutions $(T(t), I(t), V(t), B(t))$ of system (2) are nonnegative on $[0,+\infty)$ and bounded.

Proof. Let us put system (2) in a vector form by setting $Z=$ $(T, I, V, B)^{T}$ and

$$
G(Z)=\left(\begin{array}{c}
G_{1}(Z) \\
G_{2}(Z) \\
G_{3}(Z) \\
G_{3}(Z)
\end{array}\right)=\left(\begin{array}{c}
\Lambda-d T(t)-f(T(t), V(t)) V(t) \\
\int_{0}^{h_{1}} P_{1}(\tau) e^{-m_{1} \tau} f(T(t-\tau), V(t-\tau)) V(t-\tau) d \tau-\delta I(t) \\
N \delta \int_{0}^{h_{2}} P_{2}(\tau) e^{-m_{2} \tau} I(t-\tau) d \tau-c V(t)-q B(t) V(t) \\
g B(t) V(t)-\mu B(t)
\end{array}\right),
$$

where $G: C_{+} \rightarrow I R^{4}$ and $C_{+}=\left\{\phi=\left(\phi_{1}, \phi_{2}, \phi_{3}, \phi_{4}\right):\right.$ $\left.\phi \in C\left([-h, 0], I R_{+}^{4}\right)\right\}$. It is easy to check that $\left.G_{i}(Z)\right|_{Z_{i}=0} \geq 0$, $i=1,2,3,4$. Due to [14, Lemma 2], any solution of (2) with $Z(\theta) \in C_{+}$, say $Z(t)=Z(t, Z(\theta))$, is such that $Z(t) \in I R_{+}^{4}$ for all $t \geq 0$. Next we show that the solutions are also bounded. It follows from the first equation of (2) that $d T / d t \leq \Lambda-d T(t)$. This implies lim $\sup _{t \rightarrow \infty} T(t) \leq \Lambda / d$, so $T(t)$ is bounded. Let

$$
F(t)=\int_{0}^{h_{1}} P_{1}(\tau) e^{-m_{1} \tau} T(t-\tau) d \tau+I(t) .
$$


Then

$$
\begin{aligned}
\frac{d F(t)}{d t}= & \Lambda \int_{0}^{h_{1}} P_{1}(\tau) e^{-m_{1} \tau} d \tau-d \int_{0}^{h_{1}} P_{1}(\tau) e^{-m_{1} \tau} T(t-\tau) d \tau \\
& -\delta I(t) \\
\leq & \Lambda-\gamma F(t),
\end{aligned}
$$

where $\gamma=\min \{d, \delta\}$ and thus $\lim \sup _{t \rightarrow \infty} F(t) \leq \Lambda / \gamma$. This implies that $F(t)$ is bounded and so is $I(t)$. Thus, there exists a $\chi>0$ such that $I(t) \leq \chi$. It follows from the third equations in (2) that

$$
\frac{d V(t)}{d t} \leq N \delta \chi-c V(t),
$$

and consequently $V(t)$ is bounded. On the other hand, let

$$
H(t)=V(t)+\frac{q}{g} B(t) .
$$

Then,

$$
\frac{d H(t)}{d t}=N \delta \int_{0}^{h_{2}} P_{2}(\tau) e^{-m_{2} \tau} I(t-\tau) d \tau-c V(t)-\frac{q \mu}{g} B(t) .
$$

We have $d H(t) / d t \leq N \delta \chi-\xi H(t)$, where $\xi=\min \{c, \mu\}$; this implies that $H(t)$ is bounded so also for $B(t)$. Finally, all the solutions of system (2) are bounded. This completes the proof.

To simplify the notations we note that

$$
k_{1}=\int_{0}^{h_{1}} P_{1}(\tau) e^{-m_{1} \tau} d \tau, \quad k_{2}=\int_{0}^{h_{2}} P_{2}(\tau) e^{-m_{2} \tau} d \tau .
$$

Global behaviour of system (2) may depend on the basic reproduction numbers $R_{0}$ and $R_{1}$ given by

$$
R_{0}=\frac{N k_{1} k_{2} f\left(T_{0}^{*}, 0\right)}{c}
$$

where $T_{0}^{*}=\Lambda / d$ and

$$
R_{1}=\frac{N k_{1} k_{2} f(M, \mu / g)}{c},
$$

with, $M=T_{0}^{*}-\left(\mu c / d N g k_{1} k_{2}\right)$. Here, $R_{0}$ and $R_{1}$ are the basic reproduction ratios for viral infection and humoral immune response of system (2), respectively. Based on the hypotheses $\left(H_{2}\right)$ and $\left(H_{3}\right)$ it is clear that $R_{1}<R_{0}$.

\section{The Existence of Positive Equilibria}

In this section we prove the existence of positive equilibrium. The system (2) always has an infection-free equilibrium $E_{0}^{*}=$ $\left(T_{0}^{*}, 0,0,0\right)$. For other possible equilibriums, we have the following theorem.
Theorem 2. Suppose that the conditions $\left(H_{1}\right)-\left(H_{3}\right)$ are satisfied.

(1) If $R_{0}>1$, then system (2) has an infected equilibrium without $B$ cells response of the form $E_{1}^{*}=\left(T_{1}^{*}, I_{1}^{*}, V_{1}^{*}, 0\right)$ with $T_{1}^{*} \in\left(0, T_{0}^{*}\right)$.

(2) If $R_{1}>1$, then system (2) has an infected equilibrium with $B$ cells response of the form $E_{2}^{*}=\left(T_{2}^{*}, I_{2}^{*}, V_{2}^{*}, B_{2}^{*}\right)$ with $T_{2}^{*} \in(0, M)$.

Proof. The steady states of system (2) satisfy the following equations:

$$
\begin{gathered}
\Lambda-d T-f(T, V) V=0, \\
k_{1} f(T, V) V-\delta I=0, \\
N \delta k_{2} I-c V-q B V=0, \\
g B V-\mu B=0 .
\end{gathered}
$$

From the last equation of (14), we have

$$
(g V-\mu) B=0 .
$$

Equations (15) has two possible solutions, $B=0$ or $g V-\mu=0$. If $B=0,(14)_{3}$ yields $I=\left(c / N \delta k_{2}\right) V$.

By substituting this into $(14)_{2}$, we obtain that

$$
\left(k_{1} f(T, V)-\frac{c}{N k_{2}}\right) V=0,
$$

which gives $V=0$ or $k_{1} f(T, V)-\left(c / N k_{2}\right)=0$.

If $V=0$, we obtain the infection-free equilibrium $E_{0}^{*}(\Lambda / d, 0,0,0)$.

If $V \neq 0,(14)_{1}$ and $(14)_{2}$ yields

$$
I=\frac{k_{1}(\Lambda-d T)}{\delta}:=I(T) .
$$

By substituting this into $(14)_{3}$, we obtain

$$
V=\frac{N k_{1} k_{2}(\Lambda-d T)}{c}:=V(T) .
$$

Since $I \geq 0$ and $V \geq 0$, this implies that $T \leq \Lambda / d$.

Now, from $\left(H_{1}\right),\left(H_{2}\right)$, and $\left(H_{3}\right)$, the following functional

$$
K(T)=k_{1} f(T, V(T))-\frac{c}{N k_{2}},
$$

satisfies

$$
\begin{gathered}
K(0) K\left(\frac{\Lambda}{d}\right)=-\left(\frac{c}{N k_{2}}\right)^{2}\left(R_{0}-1\right)<0, \quad \text { for } R_{0}>1, \\
\dot{K}(T)=k_{1} \frac{\partial f}{\partial T}-\frac{N d k_{1} k_{2}}{c} \frac{\partial f}{\partial V}>0 .
\end{gathered}
$$

Hence, we obtain the infected equilibrium without $B$ cells response

$$
E_{1}^{*}=\left(T_{1}^{*}, I_{1}^{*}, V_{1}^{*}, 0\right)=\left(T_{1}^{*}, I\left(T_{1}^{*}\right), V\left(T_{1}^{*}\right), 0\right),
$$

where $T_{1}^{*}$ is the unique zero in $\left(0, T_{0}^{*}\right)$ of $K$ and $I$ and $V$ are given by (17) and (18). 
If $B \neq 0$, from (15), we obtain

$$
V=\frac{\mu}{g}:=V_{2}^{*},
$$

and from the first and second equation of (14), we have

$$
I=I(T)=\frac{k_{1}(\Lambda-d T)}{\delta} \geq 0 .
$$

By substituting this into $(14)_{3}$, we obtain

$$
B=B(T)=\frac{d N g k_{1} k_{2}(M-T)}{q \mu} \geq 0,
$$

which implies that $T \leq M$.

Now, from $(14)_{1}$ the functional

$$
L(T):=\Lambda-d T-f\left(T, V_{2}^{*}\right) V_{2}^{*}=0
$$

satisfies

$$
\begin{gathered}
L(0) L(M)=\Lambda\left(\frac{\mu c}{N g k_{1} k_{2}}\right)\left(1-R_{1}\right)<0, \quad \text { for } R_{1}>1 \\
\dot{L}(T)=-\frac{\mu}{g} \frac{\partial f}{\partial T}-d<0 .
\end{gathered}
$$

Hence, we obtain the infected equilibrium with $B$ cells response $E_{2}^{*}=\left(T_{2}^{*}, I\left(T_{2}^{*}\right), V_{2}^{*}, B\left(T_{2}^{*}\right)\right)$, where $T_{2}^{*}$ is the unique zero of $L$ in $(0, M)$ and $I$ and $B$ are given by (23) and (24), respectively. This completes the proof.

Remark 3. From (19) we have $K(M)=\left(c / N k_{2}\right)\left(R_{1}-1\right) \leq 0$ if $R_{1} \leq 1$. So, as $K$ is increasing in the interval $[o, \Lambda / d]$, we deduces that $M \leq T_{1}^{*}$ and consequently $V_{1}^{*}-(\mu / g) \leq 0$.

\section{Global Stability of \\ the Infection-Free Equilibrium}

In this section, we study the global stability of the infectionfree equilibrium $E_{0}^{*}$ of system (2).

Theorem 4. Suppose that the conditions $\left(H_{1}\right)-\left(H_{3}\right)$ are satisfied. Then the infection-free equilibrium $E_{0}^{*}$ of system (2) is globally asymptotically stable if $R_{0} \leq 1$.

Proof. Define a Lyapunov functional:

$$
\begin{aligned}
U_{0}(t)= & \int_{T_{0}^{*}}^{T}\left(1-\frac{f\left(T_{0}^{*}, 0\right)}{f(\sigma, 0)}\right) d \sigma+\frac{1}{k_{1}} I+\frac{1}{N k_{1} k_{2}} V \\
& +\frac{q}{N g k_{1} k_{2}} B+\frac{1}{k_{1}} \int_{0}^{h_{1}} P_{1}(\tau) e^{-m_{1} \tau} \\
& \times \int_{t-\tau}^{t} f(T(\sigma), V(\sigma)) V(\sigma) d \sigma d \tau \\
& +\frac{\delta}{k_{1} k_{2}} \int_{0}^{h_{2}} P_{2}(\tau) e^{-m_{2} \tau} \int_{t-\tau}^{t} I(\sigma) d \sigma d \tau,
\end{aligned}
$$

where $k_{1}$ and $k_{2}$ are given in (11).
It is obvious that $U_{0}$ is defined and continuously differentiable for all $T, I, V, \mathrm{~B}>0$, and $U_{0}=0$ at $E_{0}^{*}$. The time derivative of $U_{0}(t)$ along the solutions of system (2) is given by

$$
\begin{aligned}
\dot{U}_{0}= & \left(1-\frac{f\left(T_{0}^{*}, 0\right)}{f(T, 0)}\right)(\Lambda-d T-f(T, V) V) \\
& +\frac{1}{k_{1}} \int_{0}^{h_{1}} P_{1}(\tau) e^{-m_{1} \tau} f\left(T_{\tau}, V_{\tau}\right) V_{\tau} d \tau-\frac{\delta}{k_{1}} I \\
& +\frac{1}{N k_{1} k_{2}}\left(N \delta \int_{0}^{h_{2}} P_{2}(\tau) e^{-m_{2} \tau} I_{\tau} d \tau-c V-q B V\right) \\
& +\frac{q}{N g k_{1} k_{2}}(g B V-\mu B) \\
& +\frac{1}{k_{1}} \int_{0}^{h_{1}} P_{1}(\tau) e^{-m_{1} \tau}\left(f(T, V) V-f\left(T_{\tau}, V_{\tau}\right) V_{\tau}\right) d \tau \\
& +\frac{\delta}{k_{1} k_{2}} \int_{0}^{h_{2}} P_{2}(\tau) e^{-m_{2} \tau}\left(I-I_{\tau}\right) d \tau
\end{aligned}
$$

with $T_{\tau}=T(t-\tau), T=T(t), I_{\tau}=I(t-\tau), I=I(t), V_{\tau}=$ $V(t-\tau), V=V(t)$, and $B=B(t)$.

At $E_{0}^{*}$, using $\Lambda=d T_{0}^{*}$, we obtain

$$
\begin{aligned}
\dot{U}_{0}= & \left(1-\frac{f\left(T_{0}^{*}, 0\right)}{f(T, 0)}\right)\left(d T_{0}^{*}-d T\right)+f(T, V) V \frac{f\left(T_{0}^{*}, 0\right)}{f(T, 0)} \\
& -\frac{c}{N k_{1} k_{2}} V-\frac{\mu q}{N g k_{1} k_{2}} B \\
= & d\left(1-\frac{f\left(T_{0}^{*}, 0\right)}{f(T, 0)}\right)\left(T_{0}^{*}-T\right)+\frac{c}{N k_{1} k_{2}} \\
& \times\left(\frac{f(T, V)}{f(T, 0)} \frac{N k_{1} k_{2} f\left(T_{0}^{*}, 0\right)}{c}-1\right) V-\frac{\mu q}{N g k_{1} k_{2}} B .
\end{aligned}
$$

From $\left(\mathrm{H}_{2}\right)$ and $\left(\mathrm{H}_{3}\right)$ we have, respectively,

$$
\begin{aligned}
\left(1-\frac{f\left(T_{0}^{*}, 0\right)}{f(T, 0)}\right)\left(T_{0}^{*}-T\right) \leq 0 \\
\frac{f(T, V)}{f(T, 0)} \frac{N k_{1} k_{2} f\left(T_{0}^{*}, 0\right)}{c} \leq \frac{f(T, 0)}{f(T, 0)} \frac{N k_{1} k_{2} f\left(T_{0}^{*}, 0\right)}{c} \\
=\frac{N k_{1} k_{2} f\left(T_{0}^{*}, 0\right)}{c}=R_{0} .
\end{aligned}
$$

Then, $R_{0} \leq 1$ ensures that $\dot{U}_{0} \leq 0$, for all $T, I, V, B \geq 0$, $\dot{U}_{0}=0$ holds only for $T=T_{0}^{*}, V=B=0$, and from $(2)_{2}$ we obtain $I=0$. It follows that $\left\{E_{0}^{*}\right\}$ is the largest invariant set in $\left\{(T, I, V, B) \mid \dot{U}_{0}=0\right\}$. It follows from LaSalle invariance principle [15] that the infection-free equilibrium $E_{0}^{*}$ is globally asymptotically stable. 


\section{Global Stability of the Infected Equilibria}

In this section, we study the global stability of the infected equilibrium without $B$ cells response $E_{1}^{*}$ and the infected equilibrium with $B$ cells response $E_{2}^{*}$ of system (2) by the Lyapunov direct method.

We set

$$
g(x)=x-1-\ln x, \quad \text { for } x \in(0, \infty) .
$$

It is clear that for any $x>0, g(x) \geq 0$ and $g(x)$ has the global minimum $x=1$, with $g(1)=0$.

Theorem 5. Suppose that the conditions $\left(H_{1}\right)-\left(H_{4}\right)$ are satisfied. Then the equilibrium $E_{1}^{*}$ is globally asymptotically stable if $R_{1} \leq 1<R_{0}$.

Proof. Define a Lyapunov functional

$$
U_{1}=W_{1}+W_{2}
$$

where

$$
\begin{aligned}
W_{1}= & \int_{T_{1}^{*}}^{T}\left(1-\frac{f\left(T_{1}^{*}, V_{1}^{*}\right)}{f\left(\sigma, V_{1}^{*}\right)}\right) d \sigma+\frac{1}{k_{1}} I_{1}^{*} g\left(\frac{I}{I_{1}^{*}}\right) \\
& +\frac{V_{1}^{*}}{N k_{1} k_{2}} g\left(\frac{V}{V_{1}^{*}}\right)+\frac{q}{N g k_{1} k_{2}} B, \\
W_{2}= & \frac{1}{k_{1}} f\left(T_{1}^{*}, V_{1}^{*}\right) V_{1}^{*} \int_{0}^{h_{1}} P_{1}(\tau) e^{-m_{1} \tau} \\
& \times \int_{t-\tau}^{t} g\left(\frac{f(T(\sigma), V(\sigma)) V(\sigma)}{f\left(T_{1}^{*}, V_{1}^{*}\right) V_{1}^{*}}\right) d \sigma d \tau \\
& +\frac{\delta}{k_{1} k_{2}} I_{1}^{*} \int_{0}^{h_{2}} P_{2}(\tau) e^{-m_{2} \tau} \int_{t-\tau}^{t} g\left(\frac{I(\sigma)}{I_{1}^{*}}\right) d \sigma d \tau,
\end{aligned}
$$

where $k_{1}$ and $k_{2}$ are given in (11).

The function $U: T \rightarrow \int_{T_{1}^{*}}^{T}\left(1-f\left(T_{1}^{*}, V_{1}^{*}\right) / f\left(\sigma, V_{1}^{*}\right)\right) d \sigma$ verifies

$$
\dot{U}(T)=1-\frac{f\left(T_{1}^{*}, V_{1}^{*}\right)}{f\left(T, V_{1}^{*}\right)} .
$$

From $\left(H_{2}\right)$, we have $\dot{U}(T)<0$ for $T \in\left(0, T_{1}^{*}\right), \dot{U}(T)>0$ for $T \in\left(T_{1}^{*}, \infty\right)$, and $\dot{U}\left(T_{1}^{*}\right)=0$, so $U(T) \geq 0$. Consequently $U_{1}$ is nonnegative defined with respect to the endemic equilibrium $E_{1}^{*}$, which is a global minimum.
We now prove that the time derivative of $U_{1}$ is nonpositive. Calculating the time derivative of $W_{1}$ along the positive solutions of (2), we obtain

$$
\begin{aligned}
\dot{W}_{1}= & \left(1-\frac{f\left(T_{1}^{*}, V_{1}^{*}\right)}{f\left(T, V_{1}^{*}\right)}\right) \dot{T}+\frac{1}{k_{1}}\left(1-\frac{I_{1}^{*}}{I}\right) \dot{I} \\
& +\frac{1}{N k_{1} k_{2}}\left(1-\frac{V_{1}^{*}}{V}\right) \dot{V}+\frac{q}{N g k_{1} k_{2}} \dot{B} \\
= & \left(1-\frac{f\left(T_{1}^{*}, V_{1}^{*}\right)}{f\left(T, I_{1}^{*}, V_{1}^{*}\right)}\right)(\Lambda-d T-f(T, V) V) \\
& +\frac{1}{k_{1}}\left(1-\frac{I_{1}^{*}}{I}\right) \\
& \times\left(\int_{0}^{h_{1}} P_{1}(\tau) e^{-m_{1} \tau} f\left(T_{\tau}, V_{\tau}\right) V_{\tau} d \tau-\delta I\right) \\
& +\frac{1}{N k_{1} k_{2}}\left(1-\frac{V_{1}^{*}}{V}\right) \\
& \times\left(N \delta \int_{0}^{h_{2}} P_{2}(\tau) e^{-m_{2} \tau} I_{\tau} d \tau-c V-q B V\right) \\
& +\frac{q}{N g k_{1} k_{2}}(g B V-\mu B) .
\end{aligned}
$$

At $E_{1}^{*}$, by using $\Lambda=d T_{1}^{*}+f\left(T_{1}^{*}, V_{1}^{*}\right) V_{1}^{*}$ and $c=N \delta k_{2} I_{1}^{*} / V_{1}^{*}$ and $\delta / k_{1} I_{1}^{*}=f\left(T_{1}^{*}, V_{1}^{*}\right) V_{1}^{*}$, we have

$$
\begin{aligned}
\dot{W}_{1}= & \left(1-\frac{f\left(T_{1}^{*}, V_{1}^{*}\right)}{f\left(T, V_{1}^{*}\right)}\right)\left(d T_{1}^{*}-d T\right) \\
& +f\left(T_{1}^{*}, V_{1}^{*}\right) V_{1}^{*}\left(1-\frac{f\left(T_{1}^{*}, V_{1}^{*}\right)}{f\left(T, V_{1}^{*}\right)}\right) \\
& -f(T, V) V+\frac{f\left(T_{1}^{*}, V_{1}^{*}\right)}{f\left(T, V_{1}^{*}\right)} f(T, V) V \\
& +\frac{1}{k_{1}} \int_{0}^{h_{1}} P_{1}(\tau) e^{-m_{1} \tau} f\left(T_{\tau}, V_{\tau}\right) V_{\tau} d \tau-\frac{\delta}{k_{1}} I \\
& -\frac{1}{k_{1}} \frac{I_{1}^{*}}{I} \int_{0}^{h_{1}} P_{1}(\tau) e^{-m_{1} \tau} f\left(T_{\tau}, V_{\tau}\right) V_{\tau} d \tau \\
& +\frac{\delta}{k_{1}} I_{1}^{*}+\frac{\delta}{k_{1} k_{2}} \int_{0}^{h_{2}} P_{2}(\tau) e^{-m_{2} \tau} I_{\tau} d \tau-\frac{\delta I_{1}^{*}}{k_{1} V_{1}^{*}} V \\
& -\frac{\delta}{k_{1} k_{2}} \frac{V_{1}^{*}}{V} \int_{0}^{h_{2}} P_{2}(\tau) e^{-m_{2} \tau} I_{\tau} d \tau+\frac{\delta}{k_{1}} I_{1}^{*} \\
& +\frac{q}{N k_{1} k_{2}}\left(V_{1}^{*}-\frac{\mu}{g}\right) B .
\end{aligned}
$$


Calculating the time derivative of $W_{2}$, we obtain

$$
\begin{aligned}
\dot{W}_{2}= & \frac{1}{k_{1}} f\left(T_{1}^{*}, V_{1}^{*}\right) V_{1}^{*} \int_{0}^{h_{1}} P_{1}(\tau) e^{-m_{1} \tau} \\
& \times\left(\frac{f(T, V) V}{f\left(T_{1}^{*}, V_{1}^{*}\right) V_{1}^{*}}-\frac{f\left(T_{\tau}, V_{\tau}\right) V_{\tau}}{f\left(T_{1}^{*}, V_{1}^{*}\right) V_{1}^{*}}\right. \\
& \left.\quad+\ln \frac{f\left(T_{\tau}, V_{\tau}\right) V_{\tau}}{f(T, V) V}\right) d \tau \\
& +\frac{\delta}{k_{1} k_{2}} I_{1}^{*} \int_{0}^{h_{2}} P_{2}(\tau) e^{-m_{2} \tau}\left(\frac{I}{I_{1}^{*}}-\frac{I_{\tau}}{I_{1}^{*}}+\ln \frac{I_{\tau}}{I}\right) d \tau \\
= & f(T, V) V-\frac{1}{k_{1}} \int_{0}^{h_{1}} P_{1}(\tau) e^{-m_{1} \tau} f\left(T_{\tau}, V_{\tau}\right) V_{\tau} d \tau \\
& +\frac{1}{k_{1}} f\left(T_{1}^{*}, V_{1}^{*}\right) V_{1}^{*} \int_{0}^{h_{1}} P_{1}(\tau) e^{-m_{1} \tau} \ln \frac{f\left(T_{\tau}, V_{\tau}\right) V_{\tau}}{f(T, V) V} d \tau \\
& +\frac{\delta}{k_{1}} I-\frac{\delta}{k_{1} k_{2}} \int_{0}^{h_{2}} P_{2}(\tau) e^{-m_{2} \tau} I_{\tau} d \tau \\
& +\frac{\delta}{k_{1} k_{2}} I_{1}^{*} \int_{0}^{h_{2}} P_{2}(\tau) e^{-m_{2} \tau} \ln \frac{I_{\tau}}{I} d \tau .
\end{aligned}
$$

Combining (36) and (37) and by using $\left(\delta / k_{1}\right) I_{1}^{*}=$ $f\left(T_{1}^{*}, V_{1}^{*}\right) V_{1}^{*}$, we obtain

$$
\begin{aligned}
\dot{U}_{1}= & \left(1-\frac{f\left(T_{1}^{*}, V_{1}^{*}\right)}{f\left(T, V_{1}^{*}\right)}\right)\left(d T_{1}^{*}-d T\right) \\
& +f\left(T_{1}^{*}, V_{1}^{*}\right) V_{1}^{*}\left(1-\frac{f\left(T_{1}^{*}, V_{1}^{*}\right)}{f\left(T, V_{1}^{*}\right)}\right) \\
& +\frac{f\left(T_{1}^{*}, V_{1}^{*}\right)}{f\left(T, V_{1}^{*}\right)} f(T, V) V \\
& -\frac{1}{k_{1}} \frac{I_{1}^{*}}{I} \int_{0}^{h_{1}} P_{1}(\tau) e^{-m_{1} \tau} f\left(T_{\tau}, V_{\tau}\right) V_{\tau} d \tau \\
& +f\left(T_{1}^{*}, V_{1}^{*}\right) V_{1}^{*}-f\left(T_{1}^{*}, V_{1}^{*}\right) V \\
& -\frac{1}{k_{2}} \frac{f\left(T_{1}^{*}, V_{1}^{*}\right) V_{1}^{*}}{I_{1}^{*}} \frac{V_{1}^{*}}{V} \int_{0}^{h_{2}} P_{2}(\tau) e^{-m_{2} \tau} I_{\tau} d \tau \\
& +f\left(T_{1}^{*}, V_{1}^{*}\right) V_{1}^{*}+\frac{1}{k_{1}} f\left(T_{1}^{*}, V_{1}^{*}\right) V_{1}^{*} \\
& \times \int_{0}^{h_{1}} P_{1}(\tau) e^{-m_{1} \tau} \ln \frac{f\left(T_{\tau}, V_{\tau}\right) V_{\tau}}{f(T, V) V} d \tau \\
& +\frac{1}{k_{2}} f\left(T_{1}^{*}, V_{1}^{*}\right) V_{1}^{*} \int_{0}^{h_{2}} P_{2}(\tau) e^{-m_{2} \tau} \ln \frac{I_{\tau}}{I} d \tau \\
& +\frac{q}{N k_{1} k_{2}}\left(V_{1}^{*}-\frac{\mu}{g}\right) B
\end{aligned}
$$

$$
\begin{aligned}
= & \left(1-\frac{f\left(T_{1}^{*}, V_{1}^{*}\right)}{f\left(T, V_{1}^{*}\right)}\right)\left(d T_{1}^{*}-d T\right) \\
& +f\left(T_{1}^{*}, V_{1}^{*}\right) V_{1}^{*}\left(\frac{f\left(T, V_{1}^{*}\right)}{f(T, V)}-\frac{V}{V_{1}^{*}}\right) \\
& \times\left(1-\frac{f(T, V)}{f\left(T, V_{1}^{*}\right)}\right)+f\left(T_{1}^{*}, V_{1}^{*}\right) V_{1}^{*} \\
& \times\left(1-\frac{f\left(T_{1}^{*}, V_{1}^{*}\right)}{f\left(T, V_{1}^{*}\right)}+\ln \frac{f\left(T_{1}^{*}, V_{1}^{*}\right)}{f\left(T, V_{1}^{*}\right)}\right) \\
& +f\left(T_{1}^{*}, V_{1}^{*}\right) V_{1}^{*}\left(1-\frac{f\left(T, V_{1}^{*}\right)}{f(T, V)}+\ln \frac{f\left(T, V_{1}^{*}\right)}{f(T, V)}\right) \\
& +\frac{1}{k_{1}} f\left(T_{1}^{*}, V_{1}^{*}\right) V_{1}^{*} \int_{0}^{h_{1}} P_{1}(\tau) e^{-m_{1} \tau} \\
& \times\left(1-\frac{f\left(T_{\tau}, V_{\tau}\right) V_{\tau} I_{1}^{*}}{f\left(T_{1}^{*}, V_{1}^{*}\right) V_{1}^{*} I}+\ln \frac{f\left(T_{\tau}, V_{\tau}\right) V_{\tau} I_{1}^{*}}{f\left(T_{1}^{*}, V_{1}^{*}\right) V_{1}^{*} I}\right) d \tau \\
& +\frac{1}{k_{2}} f\left(T_{1}^{*}, V_{1}^{*}\right) V_{1}^{*} \int_{0}^{h_{2}} P_{2}(\tau) e^{-m_{2} \tau} g\left(\frac{I_{\tau} V_{1}^{*}}{I_{1}^{*} V}\right) d \tau \\
& +\frac{1}{k_{2}} f\left(T_{1}^{*}, V_{1}^{*}\right) V_{1}^{*} \int_{0}^{h_{2}} P_{2}(\tau) e^{-m_{2} \tau} \\
& \times\left(\frac{\mu}{g}\right) B . \\
& \times\left(1-\frac{I_{\tau} V_{1}^{*}}{I_{1}^{*} V}+\ln \frac{I_{\tau} V_{1}^{*}}{I_{1}^{*} V}\right) d \tau+\frac{q}{N k_{1} k_{2}}\left(T_{1}^{*}, V_{1}^{*}\right) V_{1}^{*} g\left(\frac{f(\tau) e_{1}^{-} m_{1} \tau}{g}\right) B\left(\frac{f\left(T_{\tau}, V_{\tau}\right) V_{\tau} I_{1}^{*}}{f\left(T, V_{1}^{*}\right)}\right)-f\left(T_{1}^{*}, V_{1}^{*}\right) V_{1}^{*} g \\
= & +\left(1-\frac{f\left(T_{1}^{*}, V_{1}^{*}\right)}{f\left(T, V_{1}^{*}\right)}\right)\left(T_{1}^{*}-T\right) \\
& +f\left(T_{1}^{*}, V_{1}^{*}\right) V_{1}^{*}\left(\frac{f\left(T, V_{1}^{*}\right)}{f(T, V)}-\frac{V}{V_{1}^{*}}\right)\left(1-\frac{f(T, V)}{f\left(T, V_{1}^{*}\right)}\right)
\end{aligned}
$$

From $\left(\mathrm{H}_{2}\right)$, we have

$$
\left(1-\frac{f\left(T_{1}^{*}, V_{1}^{*}\right)}{f\left(T, V_{1}^{*}\right)}\right)\left(T_{1}^{*}-T\right) \leq 0,
$$

and from $\left(\mathrm{H}_{3}\right)$ and $\left(\mathrm{H}_{4}\right)$ we have

$$
\left(\frac{f\left(T, V_{1}^{*}\right)}{f(T, V)}-\frac{V}{V_{1}^{*}}\right)\left(1-\frac{f(T, V)}{f\left(T, V_{1}^{*}\right)}\right) \leq 0,
$$


and as $g$ is positive, we have

$$
\dot{U}_{1} \leq \frac{q}{N k_{1} k_{2}}\left(V_{1}^{*}-\frac{\mu}{g}\right) B .
$$

From Remark 3 we have $\dot{U}_{1}(t) \leq 0$ for all $T, I, V, B \geq 0$. It is easy to verify that from (38), the largest invariant set in $\left\{(T, I, V, B) \backslash \dot{U}_{1}(t)=0\right\}$ is the singleton $\left\{E_{1}^{*}\right\}$. Using LaSalle invariance principle [15], if $R_{1} \leq 1<R_{0}$, then the equilibrium $E_{1}^{*}$ is globally asymptotically stable. This completes the proof.

Theorem 6. Suppose that the conditions $\left(H_{1}\right)-\left(H_{4}\right)$ are satisfied. Then the equilibrium $E_{2}^{*}$ is globally asymptotically stable if $R_{1}>1$.

Proof. Define a Lyapunov functional

$$
U_{2}=W_{3}+W_{4}
$$

where

$$
\begin{aligned}
W_{3}= & \int_{T_{2}^{*}}^{T}\left(1-\frac{f\left(T_{2}^{*}, V_{2}^{*}\right)}{f\left(\sigma, V_{2}^{*}\right)}\right) d \sigma+\frac{1}{k_{1}} I_{2}^{*} g\left(\frac{I}{I_{2}^{*}}\right) \\
& +\frac{V_{2}^{*}}{N k_{1} k_{2}} g\left(\frac{V}{V_{2}^{*}}\right)+\frac{q}{N g k_{1} k_{2}} B_{2}^{*} g\left(\frac{B}{B_{2}^{*}}\right), \\
W_{4}= & \frac{1}{k_{1}} f\left(T_{2}^{*}, V_{2}^{*}\right) V_{2}^{*} \int_{0}^{h_{1}} P_{1}(\tau) e^{-m_{1} \tau} \\
& \times \int_{t-\tau}^{t} g\left(\frac{f(T(\sigma), V(\sigma)) V(\sigma)}{f\left(T_{2}^{*}, V_{2}^{*}\right) V_{2}^{*}}\right) d \sigma d \tau \\
& +\frac{\delta}{k_{1} k_{2}} I_{2}^{*} \int_{0}^{h_{2}} P_{2}(\tau) e^{-m_{2} \tau} \int_{t-\tau}^{t} g\left(\frac{I(\sigma)}{I_{2}^{*}}\right) d \sigma d \tau,
\end{aligned}
$$

where $k_{1}$ and $k_{2}$ are given in (11). verifies

The function $U: T \rightarrow \int_{T_{2}^{*}}^{T}\left(1-\left(f\left(T_{2}^{*}, V_{2}^{*}\right) / f\left(\sigma, V_{2}^{*}\right)\right)\right) d \sigma$

$$
\dot{U}(T)=1-\frac{f\left(T_{2}^{*}, V_{2}^{*}\right)}{f\left(T, V_{2}^{*}\right)} .
$$

From $\left(H_{2}\right)$, we have $\dot{U}(T)<0$ for $T \in\left(0, T_{2}^{*}\right), \dot{U}(T)>0$ for $T \in\left(T_{2}^{*}, \infty\right)$, and $\dot{U}\left(T_{2}^{*}\right)=0$, so $U(T) \geq 0$. Consequently $U_{2}$ is nonnegative defined with respect to the endemic equilibrium $E_{2}^{*}$, which is a global minimum.
We now prove that the time derivative of $U_{1}$ is nonpositive. Calculating the time derivative of $W_{1}$ along the positive solutions of (2), we obtain

$$
\begin{aligned}
\dot{W}_{3}= & \left(1-\frac{f\left(T_{2}^{*}, V_{2}^{*}\right)}{f\left(T, V_{2}^{*}\right)}\right) \dot{T}+\frac{1}{k_{1}}\left(1-\frac{I_{2}^{*}}{I}\right) \dot{I} \\
& +\frac{1}{N k_{1} k_{2}}\left(1-\frac{V_{2}^{*}}{V}\right) \dot{V}+\frac{q}{N g k_{1} k_{2}}\left(1-\frac{B_{2}^{*}}{B}\right) \dot{B} \\
= & \left(1-\frac{f\left(T_{2}^{*}, V_{2}^{*}\right)}{f\left(T, I_{2}^{*}, V_{2}^{*}\right)}\right)(\Lambda-d T-f(T, V) V) \\
& +\frac{1}{k_{1}}\left(1-\frac{I_{2}^{*}}{I}\right)\left(\int_{0}^{h_{1}} P_{1}(\tau) e^{-m_{1} \tau} f\left(T_{\tau}, V_{\tau}\right) V_{\tau} d \tau-\delta I\right) \\
& +\frac{1}{N k_{1} k_{2}}\left(1-\frac{V_{2}^{*}}{V}\right)\left(N \delta \int_{0}^{h_{2}} P_{2}(\tau) e^{-m_{2} \tau} I_{\tau} d \tau\right. \\
& +\frac{q}{N g k_{1} k_{2}}\left(1-\frac{B_{2}^{*}}{B}\right)(g B V-\mu B) .
\end{aligned}
$$

At $E_{2}^{*}$, by using $\Lambda=d T_{2}^{*}+f\left(T_{2}^{*}, V_{2}^{*}\right) V_{2}^{*}, c=\left(N \delta k_{2} I_{2}^{*} / V_{2}^{*}\right)-$ $q B_{2}^{*}$, and $V_{2}^{*}=\mu / g$, we have

$$
\begin{aligned}
\dot{W}_{3}= & \left(1-\frac{f\left(T_{2}^{*}, V_{2}^{*}\right)}{f\left(T, V_{2}^{*}\right)}\right)\left(d T_{2}^{*}-d T\right) \\
& +f\left(T_{2}^{*}, V_{2}^{*}\right) V_{2}^{*}\left(1-\frac{f\left(T_{2}^{*}, V_{2}^{*}\right)}{f\left(T, V_{2}^{*}\right)}\right)-f(T, V) V \\
& +\frac{f\left(T_{2}^{*}, V_{2}^{*}\right)}{f\left(T, V_{2}^{*}\right)} f(T, V) V+\frac{1}{k_{1}} \int_{0}^{h_{1}} P_{1}(\tau) e^{-m_{1} \tau} \\
& \times f\left(T_{\tau}, V_{\tau}\right) V_{\tau} d \tau-\frac{\delta}{k_{1}} I+\frac{f\left(T_{2}^{*}, V_{2}^{*}\right)}{f\left(T, V_{2}^{*}\right)} f(T, V) V \\
& +\frac{1}{k_{1}} \int_{0}^{h_{1}} P_{1}(\tau) e^{-m_{1} \tau} f\left(T_{\tau}, V_{\tau}\right) V_{\tau} d \tau-\frac{\delta}{k_{1}} I \\
& -\frac{1}{k_{1}} \frac{I_{2}^{*}}{I} \int_{0}^{h_{1}} P_{1}(\tau) e^{-m_{1} \tau} f\left(T_{\tau}, V_{\tau}\right) V_{\tau} d \tau+\frac{\delta}{k_{1}} I_{2}^{*} \\
& +\frac{\delta}{k_{1} k_{2}} \int_{0}^{h_{2}} P_{2}(\tau) e^{-m_{2} \tau} I_{\tau} d \tau-\frac{q}{N k_{1} k_{2}} B V \\
& \frac{\delta}{k_{1} k_{2}} \frac{V_{2}^{*}}{V} \int_{0}^{h_{2}} P_{2}(\tau) e^{-m_{2} \tau} I_{\tau} d \tau+\frac{q V_{2}^{*}}{N k_{1} k_{2}} B \\
&
\end{aligned}
$$




$$
\begin{aligned}
& -\frac{1}{N k_{1} k_{2}}\left(V-V_{2}^{*}\right)\left(\frac{N \delta k_{2} I_{2}^{*}}{V_{2}^{*}}-q B_{2}^{*}\right) \\
& +\frac{q}{N k_{2} k_{2}}\left(B-B_{2}^{*}\right)\left(V-V_{2}^{*}\right) \\
& =\left(1-\frac{f\left(T_{2}^{*}, V_{2}^{*}\right)}{f\left(T, V_{2}^{*}\right)}\right)\left(d T_{2}^{*}-d T\right) \\
& +f\left(T_{2}^{*}, V_{2}^{*}\right) V_{2}^{*}\left(1-\frac{f\left(T_{2}^{*}, V_{2}^{*}\right)}{f\left(T, V_{2}^{*}\right)}\right)-f(\mathrm{~T}, V) V \\
& +\frac{f\left(T_{2}^{*}, V_{2}^{*}\right)}{f\left(T, V_{2}^{*}\right)} f(T, V) V+\frac{1}{k_{1}} \int_{0}^{h_{1}} P_{1}(\tau) e^{-m_{1} \tau} \\
& \times f\left(T_{\tau}, V_{\tau}\right) V_{\tau} d \tau-\frac{\delta}{k_{1}} I+\frac{f\left(T_{2}^{*}, V_{2}^{*}\right)}{f\left(T, V_{2}^{*}\right)} f(T, V) V \\
& +\frac{1}{k_{1}} \int_{0}^{h_{1}} P_{1}(\tau) e^{-m_{1} \tau} f\left(T_{\tau}, V_{\tau}\right) V_{\tau} d \tau-\frac{\delta}{k_{1}} I \\
& -\frac{1}{k_{1}} \frac{I_{2}^{*}}{I} \int_{0}^{h_{1}} P_{1}(\tau) e^{-m_{1} \tau} f\left(T_{\tau}, V_{\tau}\right) V_{\tau} d \tau+\frac{\delta}{k_{1}} I_{2}^{*} \\
& +\frac{\delta}{k_{1} k_{2}} \int_{0}^{h_{2}} P_{2}(\tau) e^{-m_{2} \tau} I_{\tau} d \tau-\frac{q}{N k_{1} k_{2}} B V \\
& -\frac{\delta}{k_{1} k_{2}} \frac{V_{2}^{*}}{V} \int_{0}^{h_{2}} P_{2}(\tau) e^{-m_{2} \tau} I_{\tau} d \tau+\frac{q V_{2}^{*}}{N k_{1} k_{2}} B \\
& -\frac{\delta I_{2}^{*}}{k_{1} v_{2}^{*}} V+\frac{q B_{2}^{*}}{N k_{1} k_{2}} V+\frac{\delta}{k_{1}} I_{2}^{*}-\frac{q V_{2}^{*} B_{2}^{*}}{N k_{1} k_{2}} \\
& +\frac{q}{N k_{1} k_{2}} B V-\frac{q V_{2}^{*}}{N k_{1} k_{2}} B-\frac{q B_{2}^{*}}{N k_{1} k_{2}} V+\frac{q B_{2}^{*} V_{2}^{*}}{N k_{1} k_{2}} \\
& =\left(1-\frac{f\left(T_{2}^{*}, V_{2}^{*}\right)}{f\left(T, V_{2}^{*}\right)}\right)\left(d T_{2}^{*}-d T\right) \\
& +f\left(T_{2}^{*}, V_{2}^{*}\right) V_{2}^{*}\left(1-\frac{f\left(T_{2}^{*}, V_{2}^{*}\right)}{f\left(T, V_{2}^{*}\right)}\right) \\
& -f(T, V) V+\frac{f\left(T_{2}^{*}, V_{2}^{*}\right)}{f\left(T, V_{2}^{*}\right)} f(T, V) V \\
& +\frac{1}{k_{1}} \int_{0}^{h_{1}} P_{1}(\tau) e^{-m_{1} \tau} f\left(T_{\tau}, V_{\tau}\right) V_{\tau} d \tau-\frac{\delta}{k_{1}} I \\
& -\frac{1}{k_{1}} \frac{I_{2}^{*}}{I} \int_{0}^{h_{1}} P_{1}(\tau) e^{-m_{1} \tau} f\left(T_{\tau}, V_{\tau}\right) V_{\tau} d \tau \\
& +\frac{\delta}{k_{1}} I_{2}^{*}+\frac{\delta}{k_{1} k_{2}} \int_{0}^{h_{2}} P_{2}(\tau) e^{-m_{2} \tau} I_{\tau} d \tau \\
& -\frac{\delta I_{2}^{*}}{k_{1} V_{2}^{*}} V-\frac{\delta}{k_{1} k_{2}} \frac{V_{2}^{*}}{V} \int_{0}^{h_{2}} P_{2}(\tau) e^{-m_{2} \tau} I_{\tau} d \tau+\frac{\delta}{k_{1}} I_{2}^{*} \text {. }
\end{aligned}
$$

Calculating the time derivative of $W_{4}$, we obtain

$$
\begin{aligned}
\dot{W}_{4}= & \frac{1}{k_{1}} f\left(T_{2}^{*}, V_{2}^{*}\right) V_{2}^{*} \int_{0}^{h_{1}} P_{1}(\tau) e^{-m_{1} \tau} \\
& \times\left(\frac{f(T, V) V}{f\left(T_{2}^{*}, V_{2}^{*}\right) V_{2}^{*}}-\frac{f\left(T_{\tau}, V_{\tau}\right) V_{\tau}}{f\left(T_{2}^{*}, V_{2}^{*}\right) V_{2}^{*}}\right. \\
& \left.+\ln \frac{f\left(T_{\tau}, V_{\tau}\right) V_{\tau}}{f(T, V) V}\right) d \tau \\
& +\frac{\delta}{k_{1} k_{2}} I_{2}^{*} \int_{0}^{h_{2}} P_{2}(\tau) e^{-m_{2} \tau}\left(\frac{I}{I_{2}^{*}}-\frac{I_{\tau}}{I_{2}^{*}}+\ln \frac{I_{\tau}}{I}\right) d \tau \\
= & f(T, V) V-\frac{1}{k_{1}} \int_{0}^{h_{1}} P_{1}(\tau) e^{-m_{1} \tau} f\left(T_{\tau}, V_{\tau}\right) V_{\tau} d \tau \\
& +\frac{1}{k_{1}} f\left(T_{2}^{*}, V_{2}^{*}\right) V_{2}^{*} \int_{0}^{h_{1}} P_{1}(\tau) e^{-m_{1} \tau} \ln \frac{f\left(T_{\tau}, V_{\tau}\right) V_{\tau}}{f(T, V) V} d \tau \\
& +\frac{\delta}{k_{1}} I-\frac{\delta}{k_{1} k_{2}} \int_{0}^{h_{2}} P_{2}(\tau) e^{-m_{2} \tau} I_{\tau} d \tau \\
& +\frac{\delta}{k_{1} k_{2}} I_{2}^{*} \int_{0}^{h_{2}} P_{2}(\tau) e^{-m_{2} \tau} \ln \frac{I_{\tau}}{I} d \tau .
\end{aligned}
$$

Combining (47) and (48) and by using $\left(\delta / k_{1}\right) I_{2}^{*}=f\left(T_{2}^{*}\right.$, $\left.V_{2}^{*}\right) V_{2}^{*}$, we obtain

$$
\begin{aligned}
& \dot{U}_{2}=\left(1-\frac{f\left(T_{2}^{*}, V_{2}^{*}\right)}{f\left(T, V_{2}^{*}\right)}\right)\left(d T_{2}^{*}-d T\right) \\
& +f\left(T_{2}^{*}, V_{2}^{*}\right) V_{2}^{*}\left(1-\frac{f\left(T_{2}^{*}, V_{2}^{*}\right)}{f\left(T, V_{2}^{*}\right)}\right) \\
& +\frac{f\left(T_{2}^{*}, V_{2}^{*}\right)}{f\left(T, V_{2}^{*}\right)} f(T, V) V-\frac{1}{k_{1}} \frac{I_{2}^{*}}{I} \int_{0}^{h_{1}} P_{1}(\tau) e^{-m_{1} \tau} \\
& \times f\left(T_{\tau}, V_{\tau}\right) V_{\tau} d \tau+f\left(T_{2}^{*}, V_{2}^{*}\right) V_{2}^{*} \\
& -f\left(T_{2}^{*}, V_{2}^{*}\right) V-\frac{1}{k_{2}} \frac{f\left(T_{2}^{*}, V_{2}^{*}\right) V_{2}^{*}}{I_{2}^{*}} \frac{V_{2}^{*}}{V} \\
& \times \int_{0}^{h_{2}} P_{2}(\tau) e^{-m_{2} \tau} I_{\tau} d \tau+f\left(T_{2}^{*}, V_{2}^{*}\right) V_{2}^{*} \\
& +\frac{1}{k_{1}} f\left(T_{2}^{*}, V_{2}^{*}\right) V_{2}^{*} \int_{0}^{h_{1}} P_{1}(\tau) e^{-m_{1} \tau} \\
& \times \ln \frac{f\left(T_{\tau}, V_{\tau}\right) V_{\tau}}{f(T, V) V} d \tau+\frac{1}{k_{2}} f\left(T_{2}^{*}, V_{2}^{*}\right) V_{2}^{*} \\
& \times \int_{0}^{h_{2}} P_{2}(\tau) e^{-m_{2} \tau} \ln \frac{I_{\tau}}{I} d \tau
\end{aligned}
$$




$$
\begin{aligned}
& =\left(1-\frac{f\left(T_{2}^{*}, V_{2}^{*}\right)}{f\left(T, V_{2}^{*}\right)}\right)\left(d T_{2}^{*}-d T\right) \\
& +f\left(T_{2}^{*}, V_{2}^{*}\right) V_{2}^{*}\left(\frac{f\left(T, V_{2}^{*}\right)}{f(T, V)}-\frac{V}{V_{2}^{*}}\right) \\
& \times\left(1-\frac{f(T, V)}{f\left(T, V_{2}^{*}\right)}\right)+f\left(T_{2}^{*}, V_{2}^{*}\right) V_{2}^{*} \\
& \times\left(1-\frac{f\left(T_{2}^{*}, V_{2}^{*}\right)}{f\left(T, V_{2}^{*}\right)}+\ln \frac{f\left(T_{2}^{*}, V_{2}^{*}\right)}{f\left(T, V_{2}^{*}\right)}\right) \\
& +f\left(T_{2}^{*}, V_{2}^{*}\right) V_{2}^{*}\left(1-\frac{f\left(T, V_{2}^{*}\right)}{f(T, V)}+\ln \frac{f\left(T, V_{2}^{*}\right)}{f(T, V)}\right) \\
& +\frac{1}{k_{1}} f\left(T_{2}^{*}, V_{2}^{*}\right) V_{2}^{*} \int_{0}^{h_{1}} P_{1}(\tau) e^{-m_{1} \tau} \\
& \times\left(1-\frac{f\left(T_{\tau}, V_{\tau}\right) V_{\tau} I_{2}^{*}}{f\left(T_{2}^{*}, V_{2}^{*}\right) V_{2}^{*} I}+\ln \frac{f\left(T_{\tau}, V_{\tau}\right) V_{\tau} I_{2}^{*}}{f\left(T_{2}^{*}, V_{2}^{*}\right) V_{2}^{*} I}\right) d \tau \\
& +\frac{1}{k_{2}} f\left(T_{2}^{*}, V_{2}^{*}\right) V_{2}^{*} \int_{0}^{h_{2}} P_{2}(\tau) e^{-m_{2} \tau} \\
& \times\left(1-\frac{I_{\tau} V_{2}^{*}}{I_{2}^{*} V}+\ln \frac{I_{\tau} V_{2}^{*}}{I_{2}^{*} V}\right) d \tau \\
& =d\left(1-\frac{f\left(T_{2}^{*}, V_{2}^{*}\right)}{f\left(T, V_{2}^{*}\right)}\right)\left(T_{2}^{*}-T\right)+f\left(T_{2}^{*}, V_{2}^{*}\right) V_{2}^{*} \\
& \times\left(\frac{f\left(T, V_{2}^{*}\right)}{f(T, V)}-\frac{V}{V_{2}^{*}}\right)\left(1-\frac{f(T, V)}{f\left(T, V_{2}^{*}\right)}\right) \\
& -f\left(T_{2}^{*}, V_{2}^{*}\right) V_{2}^{*} g\left(\frac{f\left(T_{2}^{*}, V_{2}^{*}\right)}{f\left(T, V_{2}^{*}\right)}\right) \\
& -f\left(T_{2}^{*}, V_{2}^{*}\right) V_{2}^{*} g\left(\frac{f\left(T, V_{2}^{*}\right)}{f(T, V)}\right) \\
& -\frac{1}{k_{1}} f\left(T_{2}^{*}, V_{2}^{*}\right) V_{2}^{*} \int_{0}^{h_{1}} P_{1}(\tau) e^{-m_{1} \tau} \\
& \times g\left(\frac{f\left(T_{\tau}, V_{\tau}\right) V_{\tau} I_{2}^{*}}{f\left(T_{2}^{*}, V_{2}^{*}\right) V_{2}^{*} I}\right) d \tau \\
& -\frac{1}{k_{2}} f\left(T_{2}^{*}, V_{2}^{*}\right) V_{2}^{*} \int_{0}^{h_{2}} P_{2}(\tau) e^{-m_{2} \tau} g\left(\frac{I_{\tau} V_{2}^{*}}{I_{2}^{*} V}\right) d \tau \text {. }
\end{aligned}
$$

From $\left(\mathrm{H}_{2}\right)$, we have

$$
\left(1-\frac{f\left(T_{2}^{*}, V_{2}^{*}\right)}{f\left(T, V_{2}^{*}\right)}\right)\left(T_{2}^{*}-T\right) \leq 0,
$$

and from $\left(H_{3}\right)$ and $\left(H_{4}\right)$ we have

$$
\left(\frac{f\left(T, V_{2}^{*}\right)}{f(T, V)}-\frac{V}{V_{2}^{*}}\right)\left(1-\frac{f(T, V)}{f\left(T, V_{2}^{*}\right)}\right) \leq 0,
$$

and as $g$ is positive, we have

$$
\dot{U}_{2} \leq 0 \text {. }
$$

Thus, the equilibrium $E_{2}^{*}$ is stable. In this case, note that $\dot{U}_{2}=$ 0 if and only if $T=T_{2}^{*}, I=I_{2}^{*}$, and $V=V_{2}^{*}$ and using the third equation of (2), we obtain $B=B_{2}^{*}$. Therefore, it follows from LaSalle's invariance principal [15] that the infected equilibrium with $B$ cells response $E_{2}^{*}$ is globally asymptotically stable. This completes the proof.

\section{Application}

In this section, we give some particular examples. In (2), if $f(T, V)=(\beta T /(1+\alpha V))$ we obtain the following model:

$$
\begin{aligned}
& \frac{d T(t)}{d t}=\Lambda-d T(t)-\frac{\beta T(t) V(t)}{1+\alpha V(t)}, \\
& \frac{d I(t)}{d t}=\int_{0}^{h_{1}} P_{1}(\tau) e^{-m_{1} \tau} \frac{\beta T(t-\tau) V(t-\tau)}{1+\alpha V(t-\tau)} d \tau-\delta I(t), \\
& \frac{d V(t)}{d t}=N \delta \int_{0}^{h_{2}} P_{2}(\tau) e^{-m_{2} \tau} I(t-\tau) d \tau \\
& \quad-c V(t)-q B(t) V(t), \\
& \frac{d B(t)}{d t}=g B(t) V(t)-\mu B(t),
\end{aligned}
$$

The global dynamics of model (53) is studied by Elaiw et al. [5]. So the work presented in [5] is a particular case of (2) because the function $(\beta T /(1+\alpha V))$ satisfies the hypothesises $\left(H_{1}\right)-\left(H_{4}\right)$.

Another particular case of (2), if $f(T, V)=(\beta T /(1+a V+$ $b T)$ ) and $h_{1}=h_{2}=\infty$, we obtain the following model which is presented by Yang et al. [13]:

$$
\begin{gathered}
\frac{d T(t)}{d t}=\Lambda-d T(t)-\frac{\beta T(t) V(t)}{1+a V(t)+b T(t)}, \\
\frac{d I(t)}{d t}=\int_{0}^{\infty} P_{1}(\tau) e^{-m_{1} \tau} \frac{\beta T(t-\tau) V(t-\tau)}{1+a V(t-\tau)+b T(t-\tau)} d \tau \\
-\delta I(t), \\
\frac{d V(t)}{d t}=N \delta \int_{0}^{\infty} P_{2}(\tau) e^{-m_{2} \tau} I(t-\tau) d \tau-c V(t) \\
-q B(t) V(t), \\
\frac{d B(t)}{d t}=g B(t) V(t)-\mu B(t) .
\end{gathered}
$$

The global asymptotic stability of possible equilibrium of (54) is established in [13].

A last example, in (2), if $f(T, V)=(\beta T /(1+\alpha V))$ and $P_{1}(\tau)=P_{2}(\tau)=\delta(\tau)$, where $\delta(\cdot)$ is the Dirac delta function, we obtain the results presented in [6]. 


\section{Conclusion}

In the current paper, we have studied an HIV-1 infection model with humoral immune response and intracellular distributed delays and general incidence rate. The model has two distributed time delays describing time needed for infection of cell and virus replication. The global stability of our model is studied by employing the method of Lyapunov functionals which are motivated by McCluskey [16] for delayed epidemic models. This general incidence represents a variety of possible incidence functions that could be used in virus dynamics model as well as epidemic models. We establish that the global dynamics are determined by two threshold parameters, the basic reproduction ratios for viral infection and humoral immune response $R_{0}$ and $R_{1}$, respectively, which depend on the incidence function and the delay. We have proved that the infection-free equilibrium $E_{0}^{*}$ is globally asymptotically stable if the basic reproduction ratios viral infection $R_{0} \leq 1$. In this case, the virus is cleared up. The hypotheses on the general incidence function are used to assure the existence of infected equilibrium without $B$ cells response $E_{1}^{*}$ and infected equilibrium with $B$ cells response $E_{2}^{*}$. We prove that if $R_{1} \leq 1<R_{0}$, the infected equilibrium without $B$ cells response $E_{1}^{*}$ is globally asymptotically stable and if $R_{1}>1$, the infected equilibrium with $B$ cells response $E_{2}^{*}$ is globally asymptotically stable.

\section{Conflict of Interests}

The authors declare that there is no conflict of interests regarding the publication of this paper.

\section{Acknowledgment}

The authors would like to thank the anonymous referees for very helpful suggestions and comments.

\section{References}

[1] A. Murase, T. Sasaki, and T. Kajiwara, "Stability analysis of pathogen-immune interaction dynamics," Journal of Mathematical Biology, vol. 51, no. 3, pp. 247-267, 2005.

[2] R. M. Anderson, R. M. May, and S. Gupta, "Non-linear phenomena in host-parasite interactions," Parasitology, vol. 99, pp. S59-S79, 1989.

[3] R. A. Arnaout, M. A. Nowak, and D. Wodarz, "HIV-1 dynamics revisited: biphasic decay by cytotoxic T lymphocyte killing?" Proceedings of the Royal Society B: Biological Sciences, vol. 267, no. 1450, pp. 1347-1354, 2000.

[4] C. Chiyaka, W. Garira, and S. Dube, "Modelling immune response and drug therapy in human malaria infection," Computational and Mathematical Methods in Medicine, vol. 9, no. 2, pp. 143-163, 2008.

[5] A. M. Elaiw, A. Alhejelan, and M. A. Alghamdi, "Global dynamics of virus infection model with antibody immune response and distributed delays," Discrete Dynamics in Nature and Society, vol. 2013, Article ID 781407, 9 pages, 2013.

[6] H.-F. Huo, Y.-L. Tang, and L.-X. Feng, "A virus dynamics model with saturation infection and humoral immunity," International Journal of Mathematical Analysis, vol. 6, no. 37-40, pp. 19771983, 2012.
[7] M. A. Nowak and C. R. M. Bangham, "Population dynamics of immune responses to persistent viruses," Science, vol. 272, no. 5258, pp. 74-79, 1996.

[8] A. S. Perelson, "Modelling viral and immune system dynamics," Nature Reviews Immunology, vol. 2, no. 1, pp. 28-36, 2002.

[9] S. Wang and D. Zou, "Global stability of in-host viral models with humoral immunity and intracellular delays," Applied Mathematical Modelling, vol. 36, no. 3, pp. 1313-1322, 2012.

[10] T. Wang, Z. Hu, F. Liao, and W. Ma, "Global stability analysis for delayed virus infection model with general incidence rate and humoral immunity," Mathematics and Computers in Simulation, vol. 89, pp. 13-22, 2013.

[11] X. Wang and S. Liu, "A class of delayed viral models with saturation infection rate and immune response," Mathematical Methods in the Applied Sciences, vol. 36, no. 2, pp. 125-142, 2013.

[12] D. Wodarz, R. M. May, and M. A. Nowak, "The role of antigenindependent persistence of memory cytotoxic T lymphocytes," International Immunology, vol. 12, no. 4, pp. 467-477, 2000.

[13] Y. Yang, H. Wang, Z. Hu, and F. Liao, "Global stability of in-host viral model with humoral immunity and Beddington-Deangelis functional response," International Journal of Life Science and Medical Research, vol. 3, no. 5, pp. 200-209, 2013.

[14] X. Yang, L. Chen, and J. Chen, "Permanence and positive periodic solution for the single-species nonautonomous delay diffusive models," Computers \& Mathematics with Applications, vol. 32, no. 4, pp. 109-116, 1996.

[15] J. P. LaSalle, The Stability of Dynamical Systems, Regional Conference Series in Applied Mathematics, SIAM, Philadelphia, Pa, USA, 1976.

[16] C. C. McCluskey, "Complete global stability for an SIR epidemic model with delay-distributed or discrete," Nonlinear Analysis: Real World Applications, vol. 11, no. 1, pp. 55-59, 2010. 


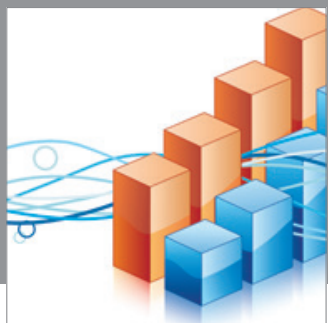

Advances in

Operations Research

mansans

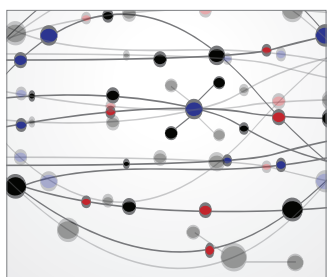

The Scientific World Journal
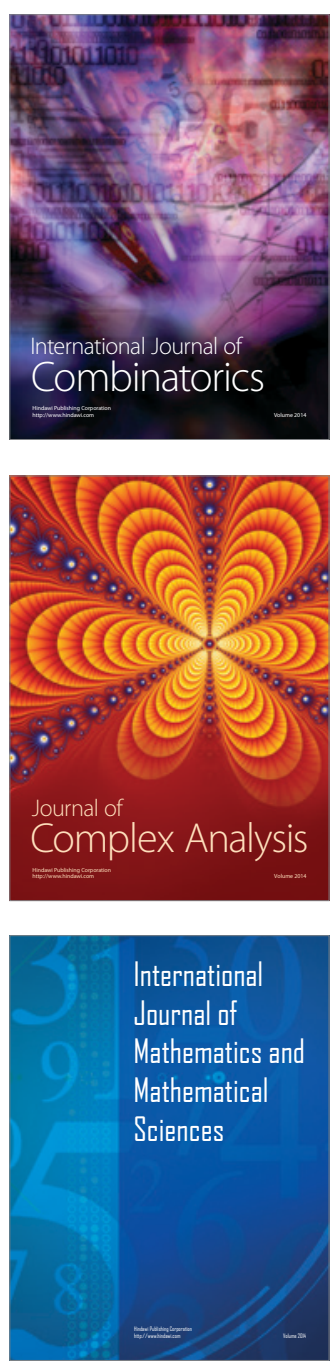
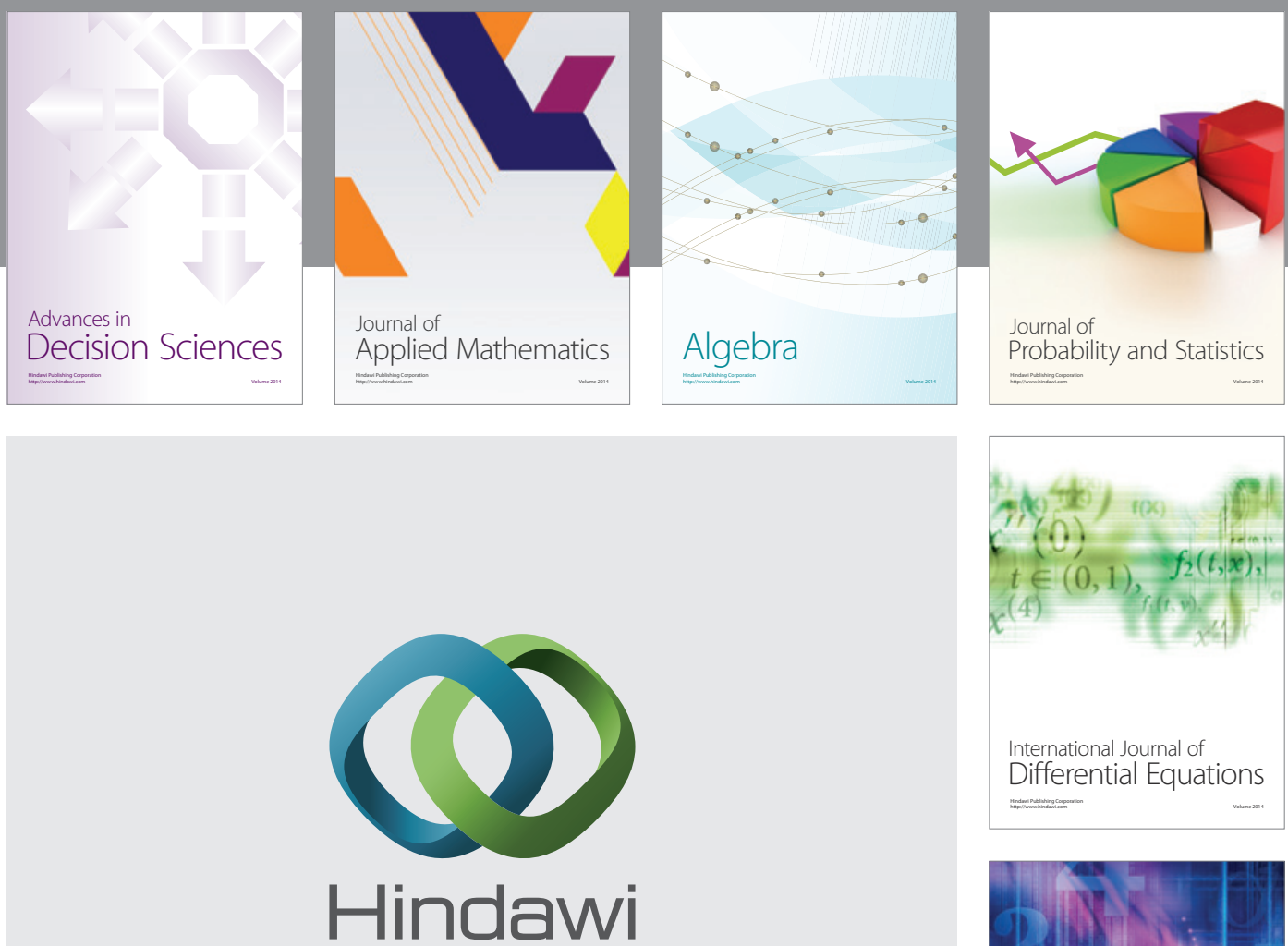

Submit your manuscripts at http://www.hindawi.com
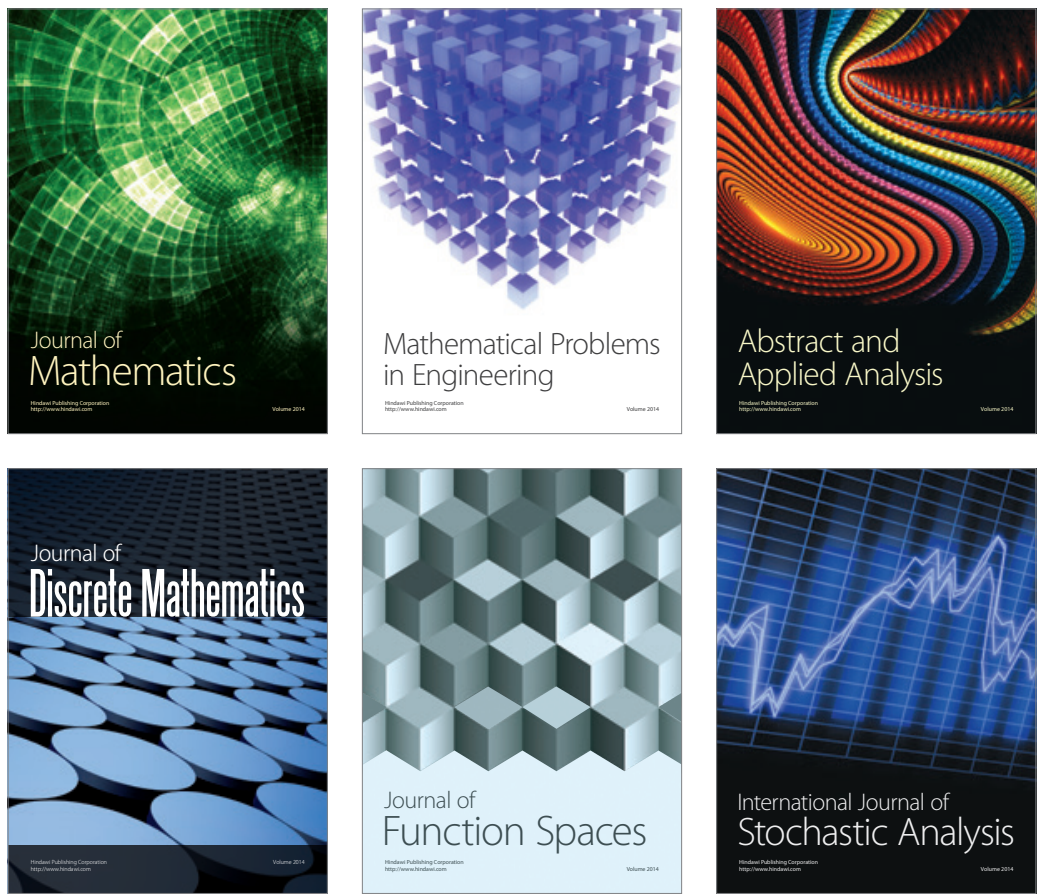

Journal of

Function Spaces

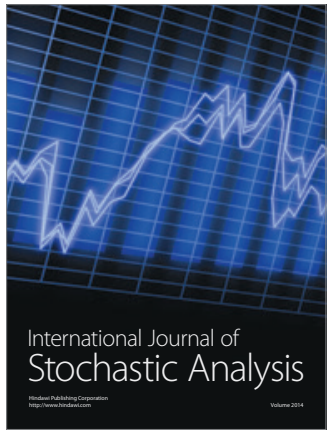

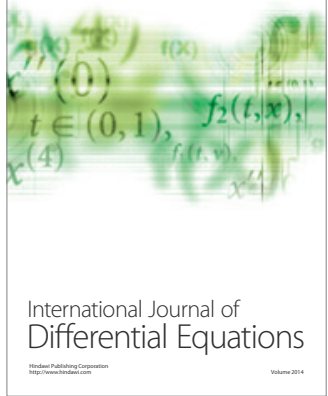
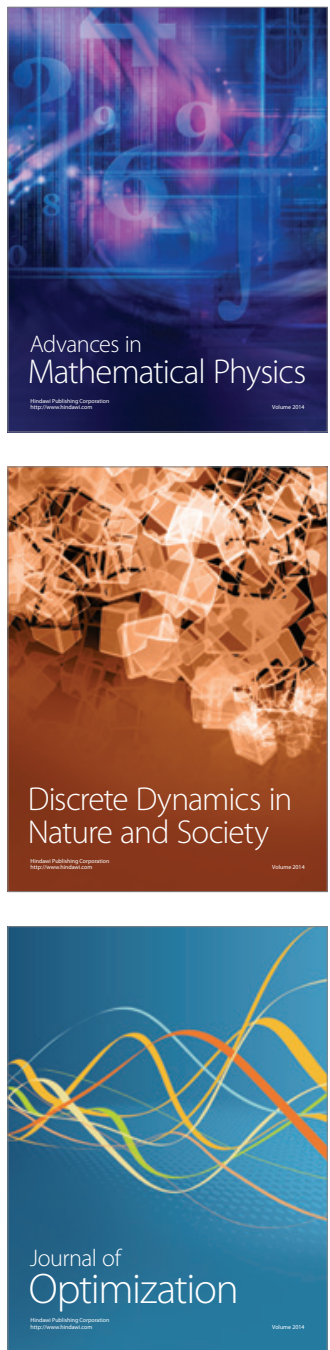